\begin{abstract}
Submitted: 30.12.2019 Accepted: 03.02.2020

Published: 31.03.2020
\end{abstract}

Keywords military personnel, tendons, sports medicine, musculoskeletal system

\section{The interrater reliability of ultrasonography for Achilles tendon structure}

\author{
Marc Paantjens ${ }^{1}$, Marco Leeuw², Pieter Helmhout ${ }^{1}$, \\ Amanda Isaac ${ }^{3}$, Michel De Maeseneer ${ }^{4}$ \\ ${ }^{1}$ Department of Military Sports Medicine, Royal Netherlands Army, Utrecht, the Netherlands \\ ${ }^{2}$ Health Care Center Havelte, Joint Support Command, Havelte, the Netherlands \\ ${ }^{3}$ Guy's and St Thomas' Hospitals, London, United Kingdom \\ ${ }^{4}$ Department of Radiology, Universitair Ziekenhuis Brussel, Vrije Universiteit Brussel, Belgium \\ Correspondence: Marc Adriaan Paantjens, Heidetuin 48, 3994 PG Houten, the Netherlands; \\ tel.:0031-622245475,e-mail:marcpaantjens@gmail.com
}

DOI: $10.15557 / J \circ U .2020 .0002$

\begin{abstract}
Aim of the study: Reliable assessment of Achilles tendon structure (architecture and morphology) may help prevent clinical symptoms or progression of Achilles tendinopathy. The objective of this study was to determine the interrater reliability of musculoskeletal ultrasonography for the assessment of the mid-portion of the Achilles tendon: (1) structure, (2) anteroposterior diameter and (3) neovascularization, in asymptomatic military personnel. Material and methods: Two sonographers acquired B-mode images of the Achilles tendon mid-portion in 74 volunteers (148 tendons) in short-axis and long-axis planes to assess its structure and measure the maximum anteroposterior diameter. Power Doppler ultrasound was performed to assess neovascularization. Tendon structure and neovascularization were graded using a modified four-graded Öhberg score. Results: Weighted kappa for assessing tendon structure showed almost perfect agreement $(0.87 ; 95 \%$ CI: 0.79, 0.95). Spearman's rho showed: a very high positive interobserver correlation for tendon structure $(0.92 ; 95 \% \mathrm{CI}: 0.89,0.94)$, a very high positive interobserver correlation for the diameter measurement in the short-axis plane $(0.91$; $95 \% \mathrm{CI}: 0.87,0.93)$ and a high positive interobserver correlation in the long-axis plane $(0.87$; 95\% CI: $0.83,0.91)$. The Wilcoxon signed-rank test showed no significant differences between observers during diameter measurements ( $p$ value $>0.05$ ). Both sonographers reported absent neovascularization in all the subjects, resulting in overall Öhberg score of 0 . Conclusions: (1) Interrater reliability of ultrasonography for grading the mid-portion Achilles tendon structure shows almost perfect agreement, and (2) ultrasonography is highly reliable in measuring the anteroposterior diameter. (3) In a large group of asymptomatic service members neovascularization of the Achilles tendon is consistently absent.
\end{abstract}




\section{Introduction}

Achilles tendinopathy is a condition that is clinically characterized by pain, swelling and impaired function, typically related to sports or exercise overuse $\mathrm{e}^{(1,2)}$. The average age at the first presentation is 43 years $^{(3)}$. However, in highly active subjects, tendinopathy can occur at a younger age ${ }^{(4)}$. Achilles tendon disorders are common in military personnel. Individuals who are overweight, report moderate alcohol use and those with a prior diagnosis of tendinopathy are at higher risk for developing pathology ${ }^{(5)}$.

Although Achilles tendinopathy incorporates elements of the inflammatory response, it is generally considered to represent a degenerative condition ${ }^{(6,7)}$. Degenerative tendon abnormalities are common in asymptomatic individuals above the age of 35 years $^{(8)}$. In sporting populations, they have been identified in up to $59 \%$ of asymptomatic athletes $^{(7)}$. Such tendon changes are predictive of future symptomatic tendinopathy, with at least a sevenfold increased risk $^{(7)}$.

Musculoskeletal ultrasonography (MSU) is currently able to detect early changes in tendon structure (architecture and morphology) due to its excellent spatial resolution ${ }^{(9)}$. MSU-based assessment of the Achilles tendon can help in making a diagnosis or defining treatment strategy. It may also identify individuals at risk for Achilles tendinopathy and allow preventative programs to be implemented $^{(7)}$. The department of sports medicine of the Royal Netherlands Armed Forces is exploring all options to predict and reduce dropout from military training due to injuries. Therefore, we sought to determine the interrater reliability of MSU for assessing the mid-portion Achilles tendon: (1) structure (2) anteroposterior diameter and (3) neovascularization, in a cohort of asymptomatic service members.

\section{Material and methods}

This cross-sectional study was approved by the ethics committee METC Brabant, Tilburg, the Netherlands (approval number NW2018-72) and by the Joint Health Care Division of the Ministry of Defence. All participants provided written informed consent before participation. Sample size calculation was performed according to recently published guidelines ${ }^{(11)}$. Based on a default $\alpha$ of .05 and statistical power $(1-\beta)$ of .80 , a sample size of 74 subjects was calculated. In January and February 2019, asymptomatic service members were recruited from an infantry unit in the Royal Netherlands Army. Men and women in the age range of $18-58$ years were eligible for inclusion. The exclusion criteria were: (1) an episode of Achilles tendinopathy within the last 12 months, (2) prior Achilles tendon surgery, (3) rheumatoid arthritis, diabetes mellitus, psoriasis or spondyloarthropathy, and (4) recent or current (at the time of recruitment to the study) use of statins, fluoroquinolones or corticosteroids ${ }^{(12)}$.
Grayscale MSU and power Doppler ultrasound (PDU) examinations were performed (Philips CX50, Philips, Eindhoven, the Netherlands) and measurements were obtained with an L12-3 MHz broadband linear-array transducer. The machine settings for MSU and PDU were standardized. A week before performing the measurements in the study participants, both sonographers (two physical therapists, MSc in MSU, with at least 5 years of experience) defined and agreed standardized consistent settings and practiced on a volunteer. All studies were performed independently by the two sonographers, blinded to each other's measurements to prevent review bias.

Short axis views followed by long axis views of the midportion of the Achilles tendon were acquired according to the "Musculoskeletal Ultrasound Technical Guidelines VI. Ankle", published online by the European Society of Musculoskeletal Radiology (ESSR) ${ }^{(13)}$.

To minimize observer bias, both tendon structure and neovascularization were graded using the modified fourgraded Öhberg score (Tab. 1) ${ }^{(10)}$.

All investigations started with grading tendon structure while subjects were lying prone on the examination table with the ankle joints flexed at $90^{\circ}$. The transducer was placed as perpendicular as possible, with a uniform layer of gel applied to minimize anisotropy artifacts and to standardize the scanning technique. The maximum anteroposterior Achilles tendon diameter was measured in the Achilles tendon mid-portion, located 2-6 centimeters proximal to the superior border of the calcaneus. At first the thickest part of the tendon was determined in short axis views. Subsequently, this part of the tendon was scanned longitudinally, and the diameter was measured in this plane. After the dimensional measures were completed, the participants were asked to relax the foot for assessment of neovascularization. Transducer pressure was as minimal as possible for this assessment. For calculating percentages of structural abnormalities, a tendon was considered structurally abnormal if one of the two sonographers graded it as Öhberg score 1-3.

The statistical analysis was performed using MedCalc ${ }^{\circledR}$ statistics (version 18.11.3). The Kolmogorov-Smirnov test was used to assess the normality of data ${ }^{(14)}$. Ordinal data (Öhberg scores for tendon structure) and ratio data (anteroposterior Achilles tendon diameter) were not normally distributed. Non-parametric tests were therefore chosen to assess the interrater reliability and differences between paired samples. The Spearman's rank correlation coefficient (Spearman`s rho: $\rho$ ) was used to calculate the correlation between raters in assessing Achilles tendon structure and measuring the anteroposterior tendon diameter ${ }^{(15)}$. Interrater reliability for evaluation of the Achilles tendon structure was calculated as weighted Kappa-coefficient $(\mathrm{kw})^{(16)}$. The Wilcoxon signed-rank test was applied to evaluate quantitative differences between sonographers in measuring the tendon diameter. 
Tab. 1. The modified four-graded Öhberg score for Achilles tendon structure and neovascularization ${ }^{10}$

\begin{tabular}{|c|l|l|}
\hline & \multicolumn{1}{|c|}{ Öhberg score for Achilles tendon structure } & \multicolumn{1}{c|}{ Öhberg score for neovascularization } \\
\hline 0 & Normal structure (homogeneous echogenicity) & No neovascularization \\
\hline 1 & Light structural changes (discrete hypoechogenic areas) & Mild neovascularization (a few solitary blood vessels) \\
\hline 2 & $\begin{array}{l}\text { Moderate structural changes } \\
\text { (some well-defined hypoechogenic areas) }\end{array}$ & $\begin{array}{l}\text { Moderate neovascularization } \\
\text { (moderate quantity, most transversal blood vessels) }\end{array}$ \\
\hline 3 & Severe structural changes (extended hypoechogenic areas) & $\begin{array}{l}\text { Severe neovascularization (several, mostly horizontal blood vessels } \\
\text { spread in the whole depth of the tendon) }\end{array}$ \\
\hline
\end{tabular}

Tab. 2. Demographics ( $\mathrm{n}=74,148$ Achilles tendons)

\begin{tabular}{|l|c|c|}
\hline & Mean* $^{*}$ & Range \\
\hline Age (years) & $33 \pm 11.7$ & $18-59$ \\
\hline Height $(\mathrm{cm})$ & $183 \pm 6.2$ & $164-199$ \\
\hline Weight $(\mathrm{kg})$ & $85 \pm 10.7$ & $63-120$ \\
\hline BMI & $25.4 \pm 2,3$ & $21-31$ \\
\hline *Values are mean \pm SD \\
\hline
\end{tabular}

Tab. 3. The distribution of the pattern of the Achilles tendon structure for two observers using the Öhberg score $(\mathrm{n}=74,148$ Achilles tendons)

\begin{tabular}{|c|c|c|c|c|}
\hline & \multicolumn{2}{|c|}{ Observer 1} & \multicolumn{2}{|c|}{ Observer 2} \\
\hline $\begin{array}{l}\text { Öhberg } \\
\text { score }\end{array}$ & $\begin{array}{c}\text { Tendons } \\
(n=)\end{array}$ & $\begin{array}{l}\% \text { of } 148 \\
\text { tendons }\end{array}$ & $\begin{array}{c}\text { Tendons } \\
(n=)\end{array}$ & $\begin{array}{l}\% \text { of } 148 \\
\text { tendons }\end{array}$ \\
\hline 0 & 120 & 81.1 & 124 & 83.8 \\
\hline 1 & 23 & 15.5 & 18 & 12.2 \\
\hline 2 & 3 & 1.4 & 6 & 4.1 \\
\hline 3 & 2 & 1.4 & 0 & 0.0 \\
\hline Total & 148 & 100 & 148 & 100 \\
\hline \multicolumn{5}{|c|}{$n-$ number of tendons } \\
\hline
\end{tabular}

Tab. 4. MSU measurements of the Achilles tendon anteroposterior diameter $(\mathrm{n}=74,148$ Achilles tendons)

\begin{tabular}{|c|c|c|c|c|}
\hline & \multicolumn{2}{|c|}{ Short axis } & \multicolumn{2}{|c|}{ Long axis } \\
\hline & $\begin{array}{c}\text { Thickness } \\
(\mathrm{mm}) \\
\text { Mean* }^{*}\end{array}$ & $\begin{array}{l}\text { Range }(\mathrm{mm}) \\
\text { Min-Max }\end{array}$ & $\begin{array}{c}\begin{array}{c}\text { Thickness } \\
(\mathrm{mm})\end{array} \\
\text { Mean* }^{*}\end{array}$ & $\begin{array}{l}\text { Range }(\mathrm{mm}) \\
\text { Min-Max }\end{array}$ \\
\hline Observer 1 & $5.0(4.9,5.2)$ & $3.7-10.6$ & $5.0(4.8,5.2)$ & $3.5-11.4$ \\
\hline Observer 2 & $5.1(4.9,5.2)$ & $3.7-10.9$ & $5.0(4.8,5.1)$ & $3.2-11.1$ \\
\hline Combined & $5.1(4.9,5.2)$ & $3.7-10.9$ & $5.0(4.9,5.1)$ & $3.2-11.4$ \\
\hline
\end{tabular}

Bland-Altman plots were constructed to represent the interrater agreement ${ }^{(17)}$.

\section{Results}

Seventy-four infantry soldiers (all men) were included in the study. Demographic data are presented in Table 2. In total, 148 tendons (74 participants) were evaluated of which 28 tendons (18.9\%) showed signs of degeneration, and were subsequently graded as abnormal (Öhberg score 1 or more). In participants under the age of 35 years (47 participants, 94 tendons), 10 tendons (11\%) were graded as abnormal. Most abnormalities were seen in asymptomatic service members of 35 years and older. In this age group (27 participants, 54 tendons), 18 Achilles tendons (33.3\%) were considered abnormal. Bilateral Achilles abnormalities were present in 9 out of 74 participants $(12 \%)$.

Both sonographers reported that none of the service members showed signs of Achilles tendon neovascularization on PDU, resulting in overall Öhberg scores of 0 . The distribution of the pattern of the observed Achilles tendon structure is presented in Table 3. Measurements of the Achilles tendon anteroposterior diameter are shown in Table 4.

Weighted Kappa for assessing the Achilles tendon structure showed almost perfect agreement (kw 0.87; 95\% CI: $0.79,0.95)$. Spearman's rho showed: (1) a very high positive interobserver correlation for Achilles tendon structure ( $\rho 0.92 ; 95 \%$ CI: $0.89,0.94),(2)$ a very high positive interobserver correlation for the anteroposterior diameter measurement in the short-axis plane ( $0.91 ; 95 \%$ CI: $0.87,0.93)$ and (3) a high positive correlation in the LA plane ( $\rho 0.87 ; 95 \%$ CI: $0.83,0.91$ ). The Wilcoxon signed-rank test showed no significant differences $(=p$-value $>0.05)$ between the observers for both long-axis $(p=0.073)$ and short-axis $(p=0.189)$ measurements.

The Bland-Altman plot graphically visualizes the differences between observers 1 and 2 in measuring the shortaxis Achilles tendon diameter (Fig. 1) and long-axis tendon diameter (Fig. 2). The paired differences are plotted against the mean difference for both observers. The mean difference between observers was $-0.004 \mathrm{~cm}(95 \% \mathrm{CI}:-0.009$, $0.001)$ for measuring the short-axis tendon diameter and $0.003 \mathrm{~cm}(95 \% \mathrm{CI}:-0.002,0.008)$ for the long-axis tendon diameter.

\section{Discussion}

Asymptomatic Achilles tendon abnormalities are predictive of future symptomatic tendinopathy ${ }^{(7)}$. MSU assessment of tendon structure may help prevent clinical symptoms by identifying high-risk individuals, and allowing preventative interventions to be implemented ${ }^{(7)}$. Our results demonstrate that in asymptomatic service members, MSU shows almost perfect agreement and a very high interobserver correlation in grading Achilles tendon structure using the Öhberg score. These results differ from Sunding et al. ${ }^{(10)}$, who reported a poor to moderate 


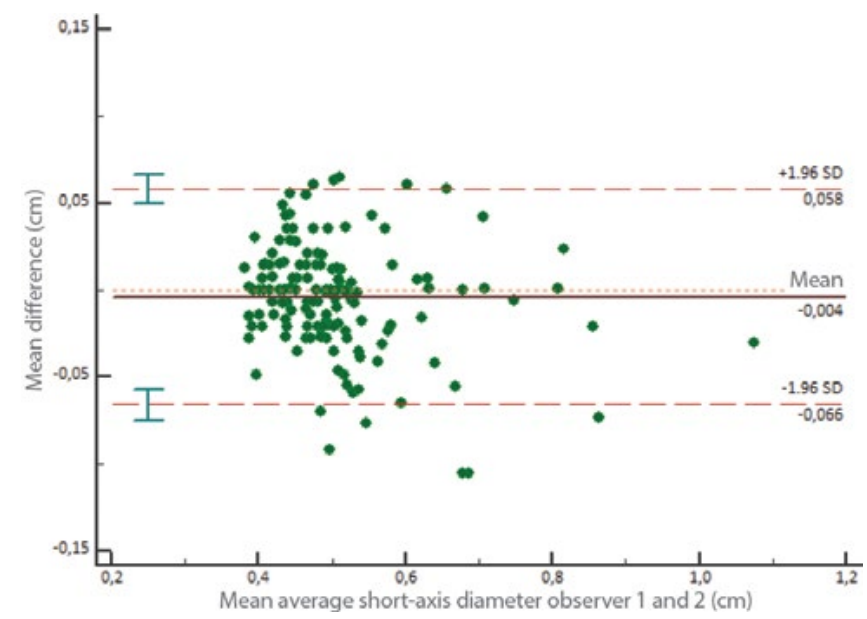

Fig. 1. The Bland-Altman plot graphically visualizes the differences between observers 1 and 2 in measuring the Achilles tendon short-axis diameter. The paired differences are plotted against the mean difference for both observers (solid horizontal black line). The two broken red lines represent the upper and lower 95\% limit of agreement (LOA). The mean difference between observers was $-0.004 \mathrm{~cm}(95 \% \mathrm{CI}:-0.009,0.001)$, the lower LOA was $-0.066 \mathrm{~cm}(95 \% \mathrm{CI}:-0.075,-0.057)$ and the upper LOA was $0.058 \mathrm{~cm}(95 \%$ CI: 0.049, 0.067). SD - standard deviation; LOA - limits of agreement; CI - confidence interval

interobserver reliability and a weak to moderate interobserver correlation for grading Achilles tendon structure ${ }^{(10)}$. The different findings of these two studies may be attributed to different study populations. We evaluated a cohort of asymptomatic participants, while Sunding et al. ${ }^{(10)}$ recruited both symptomatic and asymptomatic individuals. A larger variation in tendon architecture among the symptomatic participants could have contributed to the lower interobserver agreement and correlation. Most Achilles tendons in our study were graded with Öhberg scores 0 and 1, whilst Öhberg scores 2 and 3 were found to be rare (Tab. 3).

Furthermore, in our study both sonographers were equally experienced, while Sunding et al. ${ }^{(10)}$ used observers with different experience levels.

We also found a very high positive correlation for measuring Achilles tendon anteroposterior diameter in the short-axis plane and a high positive correlation in the long-axis plane. These findings are consistent with previous studies ${ }^{(10,18)}$.

Almost $19 \%$ of all asymptomatic Achilles tendons examined in our study showed degenerative signs on MSU. In contrast, Nicol et al. ${ }^{(19)}$ reported a much higher prevalence of degenerative Achilles tendon changes in asymptomatic service members of the British Armed Forces: 59\% of all asymptomatic Achilles tendons exhibited ultrasonographic signs of degeneration. Different inclusion and exclusion criteria for service members to participate may explain the large difference in the prevalence between both studies. In our study, individuals who experienced an episode of Achilles tendinopathy within the last 12 months were excluded, while the

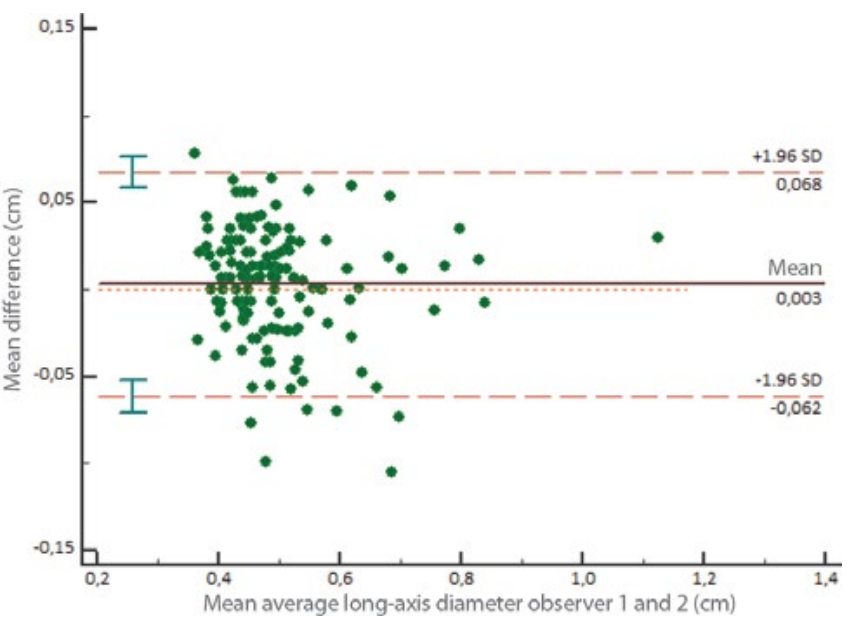

Fig. 2. The Bland-Altman plot graphically visualizes the differences between observers 1 and 2 in measuring the Achilles tendon long-axis diameter. The paired differences are plotted against the mean difference for both observers (solid horizontal black line). The two broken red lines represent the upper and lower 95\% LOA. The mean difference between observers was $0.003 \mathrm{~cm}$ (95\% CI: -0.002, 0.008), the lower LOA was $-0.06 \mathrm{~cm}(95 \%$ CI: -0.07, -0.05) and the upper LOA was $0.07 \mathrm{~cm}(95 \%$ CI: 0.06, 0.08). SD - standard deviation; $L O A$ - limits of agreement; CI-confidence interval

British authors allowed subjects with a recent episode of Achilles tendinopathy when free of symptoms for at least four weeks. Tendon degeneration on MSU appears to be more common in service members with a prior history of symptomatic Achilles tendinopathy ${ }^{(19)}$, which most likely has contributed to the higher percentage of abnormal tendons in their study. Furthermore, Nicol et al. ${ }^{(19)}$ did not mention excluding service members on the basis of various disorders or medication use, while both these factors are known to cause tendon degeneration ${ }^{(12)}$. This can also account for the higher prevalence of abnormal Achilles tendons in their study.

Achilles tendinopathy is often associated with neovascularization, but the clinical significance is somewhat contested $^{(20-22)}$. It is assumed that asymptomatic tendons show no signs of neovascularization on PDU, although some studies suggest the opposite ${ }^{(23,24)}$. In contrast, patients with symptomatic tendinopathy do not always exhibit signs of neovascularization on PDU ${ }^{(22)}$. In our current study, we did not observe neovascularization in any of the 148 asymptomatic Achilles tendons examined. From this we may conclude that this phenomenon is possibly limited to pathological tendons.

During the assessment of Achilles tendon diameter, we frequently observed that the thickest part of the Achilles tendon mid-portion was often the location where the plantaris tendon inserted on the Achilles tendon. The plantaris tendon is reported to be present in $92-94 \%$ of individu$\mathrm{als}^{(25)}$. In most cases, it inserts into the medial border of the mid-portion Achilles tendon, while in 6-8\% of individuals the tendon inserts into the flexor retinaculum ${ }^{(25)}$. In our study we observed subjects in whom the plantaris tendon was not present or visible. We also evaluated subjects 


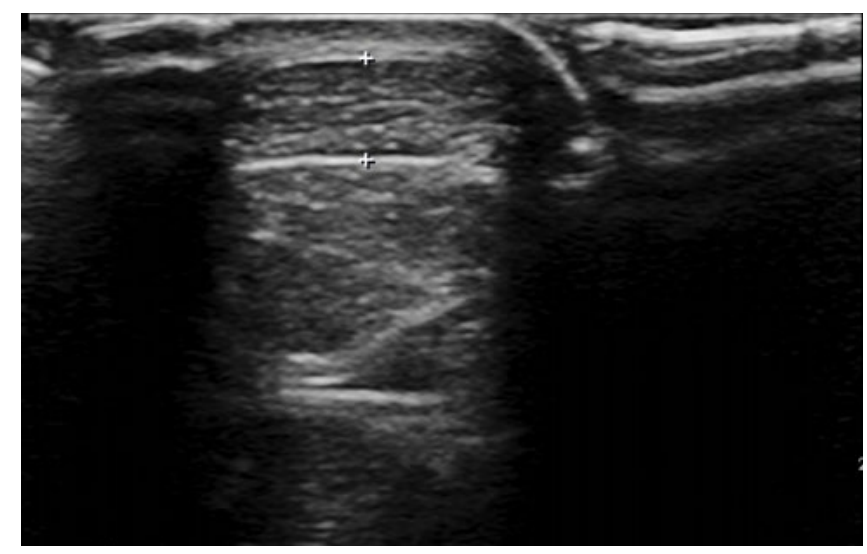

Fig. 3. MSU image of a volunteer with Öhberg score 0, short-axis view $(++$ anterior and posterior border of the Achilles tendon). The tendon structure was graded as normal with homogeneous echogenicity

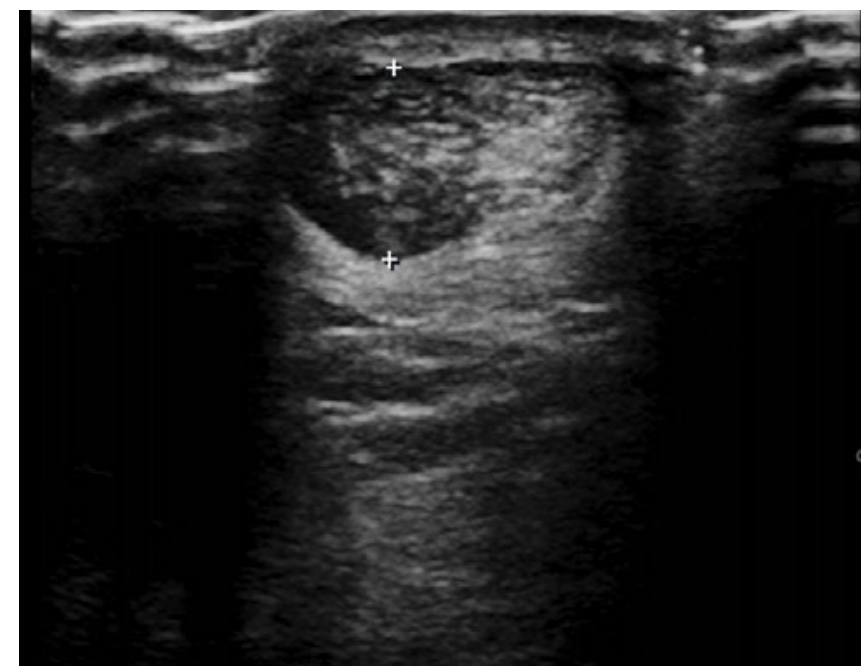

Fig. 5. Short-axis view of an MSU image in which observer 1 graded the Öhberg score as 3, while observer 2 graded the Öhberg score as $2(++$ anterior and posterior border of the Achilles tendon). Discriminating severe structural changes (extended hypoechogenic areas) from moderate structural changes (some well-defined hypoechogenic areas) turned out to be difficult in this subject

with visible plantaris tendons, in whom it was sometimes difficult to distinguish the plantaris tendon from the borders of the Achilles tendon. Therefore, when quantifying or comparing Achilles tendon diameter, sonographers should consider the locoregional anatomy, including the presence or absence of the plantaris tendon.

This study has several limitations. First, the absolute size of the anteroposterior tendon measurements may be an overestimation, since the tendon borders of the plantaris tendon and Achilles tendon were not always discernable on MSU.

Second, our study results may be limited to the male gender because we were not able to recruit any female volunteers. In the unit where we recruited participants, only 8 out of 440 service members were women. In the military

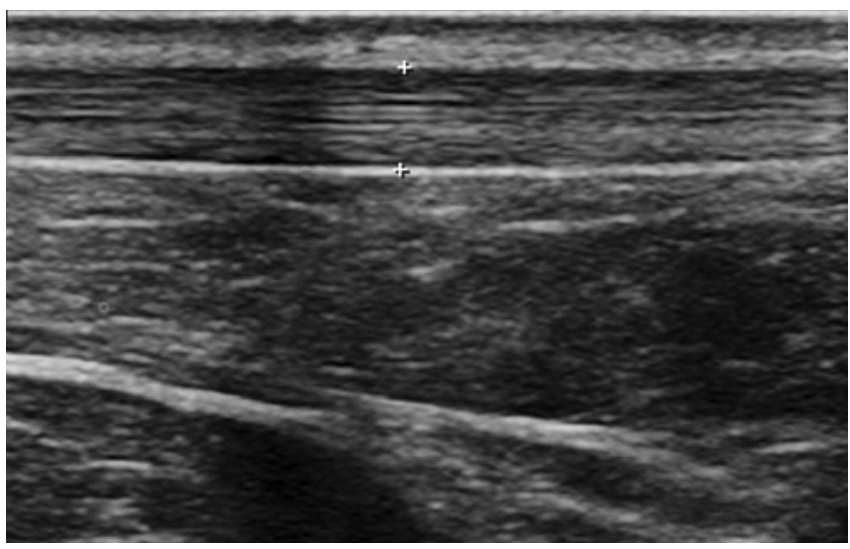

Fig. 4. MSU image of a volunteer with Öhberg score 0, long-axis view $(++$ anterior and posterior border of the Achilles tendon). The tendon structure was graded as normal with homogeneous echogenicity

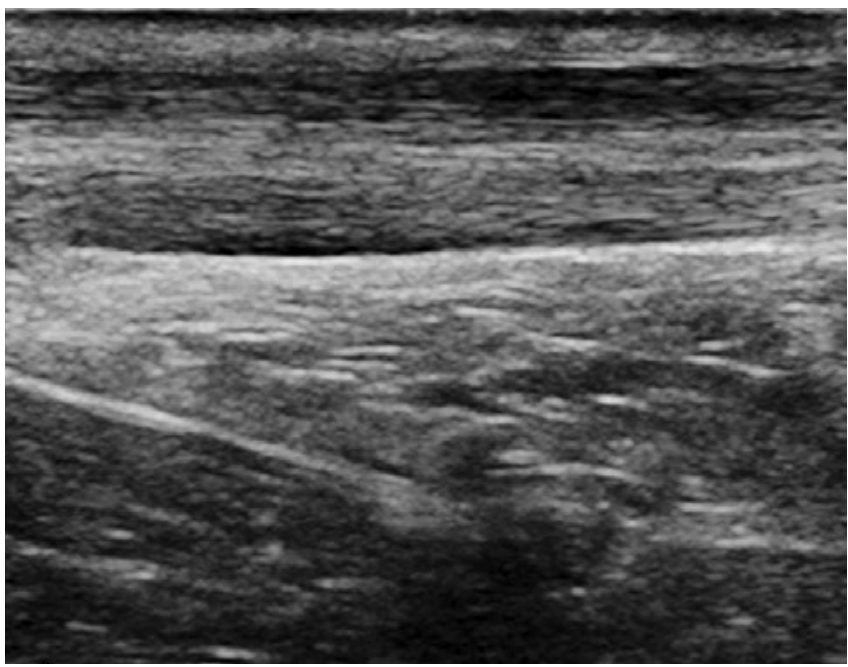

Fig. 6. Long axis view of an MSU image in which observer 1 graded the Öhberg score as 3, while observer 2 graded the Öhberg score as 2. Discriminating severe structural changes (extended hypoechogenic areas) from moderate structural changes (some well-defined hypoechogenic areas) turned out to be difficult in this subject

population, Owens et al. ${ }^{(5)}$ found no evidence that gender might be a risk factor for mid-portion Achilles tendinopathy; however, there is conflicting evidence regarding its significance in the general population.

Our high reliability scores may not be applicable to the assessment of moderate or severe Achilles tendon changes, as these changes were rare in our study population (Tab. 3). Discriminating normal tendons (Fig. 3 and Fig. 4) from abnormal tendons may be easier than judging whether a specific Achilles tendon shows moderate or severe signs of degeneration (Fig. 5 and Fig. 6). Most tendon abnormalities occurred in service members of 35 years and older, as previously reported in the literature $^{(8)}$. Increasing the minimum age for inclusion to 35 years, could result in finding more moderate or severe Achilles tendon abnormalities. 


\section{Conclusion}

In asymptomatic individuals, MSU is highly reliable for grading mid-portion Achilles tendon structure and measuring anteroposterior tendon diameter. The absence of neovascularization in our asymptomatic population suggests that this phenomenon may be limited to pathological Achilles tendons. Caution should be warranted when generalizing our reliability results to individuals with symptomatic or severe Achilles tendon changes, as these changes appear to be more difficult to grade.

\section{References}

1. Kujala UM, Sarna S, Kaprio J: Cumulative incidence of Achilles tendon rupture and tendinopathy in male former elite athletes. Clin J Sport Med 2005; 15: 133-135.

2. Maffulli N, Khan KM, Puddu G: Overuse tendon conditions: time to change a confusing terminology. Arthroscopy 1998; 14: 840-843.

3. de Jonge $\mathrm{S}$, van den Berg C, de Vos RJ, van der Heide HJ, Weir A, Verhaar JA et al.: Incidence of midportion Achilles tendinopathy in the general population. Br J Sports Med 2011; 45: 1026-1028.

4. Alfredson H, Lorentzon R: Chronic Achilles tendinosis: recommendations for treatment and prevention. Sports Med 2000; 29: 135-146.

5. Owens BD, Wolf JM, Seelig AD, Jacobson IG, Boyko EJ, Smith B et al:: Risk factors for lower extremity tendinopathies in military personnel. Orthop J Sports Med 2013; 1: 2325967113492707.

6. Cook JL, Rio E, Purdam CR, Docking SI: Revisiting the continuum model of tendon pathology: what is its merit in clinical practice and research? Br J Sports Med 2016; 50: 1187-1191.

7. McAuliffe S, McCreesh K, Culloty F, Purtill H, O'Sullivan K: Can ultrasound imaging predict the development of Achilles and patellar tendinopathy? A systematic review and meta-analysis. Br J Sports Med 2016; 50: 1516-1523.

8. Kannus P, Józsa L: Histopathological changes preceding spontaneous rupture of a tendon. A controlled study of 891 patients. J Bone Joint Surg Am 1991; 73: 1507-1525.

9. Bleakney RR, White LM: Imaging of the Achilles tendon. Foot Ankle Clin 2005; 10: 239-254.

10. Sunding K, Fahlström M, Werner S, Forssblad M, Willberg L: Evaluation of Achilles and patellar tendinopathy with greyscale ultrasound and colour Doppler: using a four-grade scale. Knee Surg Sports Traumatol Arthrosc 2016; 24: 1988-1996.

11. Bujang MA, Baharum N: Guidelines of the minimum sample size requirements for Cohen's Kappa. Epidemiolog Biostat Public Health 2017; 14: 1-10.

12. Scott A, Backman LJ, Speed C: Tendinopathy: Update on pathophysiology. J Orthop Sports Phys Ther 2015; 45: 833-841.

13. Beggs I, Bianchi S, Bueno A, Cohen M, Court-Payen M, Grainger A et al.: Musculoskeletal ultrasound technical guidelines VI. Ankle. Online: https://essr.org/content-essr/uploads/2016/10/ankle.pdf (Accessed: 29.12.2019).

\section{Conflict of interest}

The authors declare that they have no conflict of interest.

\section{Acknowledgement}

We thank the staff and personnel of the Johan Willem Friso 44th mechanized infantry battalion (Royal Netherlands Army) for participating in this study.
14. Marsaglia G, Tsang WW, Wang J: Evaluating Kolmogorov's distribution. J Stat Soft 2003; 8: 1-4.

15. Bishara AJ, Hittner JB: Testing the significance of a correlation with nonnormal data: Comparison of Pearson, Spearman, transformation, and resampling approaches. Psychol Methods 2012; 17: 399-417.

16. Viera AJ, Garrett JM: Understanding interobserver agreement: the kappa statistic. Fam Med 2005; 37: 360-363.

17. Bland JM, Altman DG: Agreed statistics: measurement method comparison. Anesthesiology 2012: 116: 182-185.

18. Johannsen F, Jensen S, Stallknecht SE, Olsen LO, Magnusson SP: Sonographic measurements of the achilles tendon, plantar fascia, and heel fat pad are reliable: A test-retest intra- and intertester study. J Clin Ultrasound 2016; 44: 480-486.

19. Nicol AM, McCurdie I, Etherington J: Use of ultrasound to identify chronic Achilles tendinosis in an active asymptomatic population. J R Army Med Corps 2006; 152: 212-216.

20. de Jonge S, Warnaars JL, de Vos RJ, Weir A, van Schie HT, Bierma-Zeinstra SM et al.: Relationship between neovascularization and clinical severity in Achilles tendinopathy in 556 paired measurements. Scand J Med Sci Sports 2014; 24: 773-778.

21. De Marchi A, Pozza S, Cenna E, Cavallo F, Gays G, Simbula L et al.: In Achilles tendinopathy, the neovascularization, detected by contrastenhanced ultrasound (CEUS), is abundant but not related to symptoms. Knee Surg Sports Traumatol Arthrosc 2018; 26: 2051-2058.

22. Yang X, Pugh ND, Coleman DP, Nokes LD: Are Doppler studies a useful method of assessing neovascularisation in human Achilles tendinopathy? A systematic review and suggestions for optimizing machine settings. J Med Eng Technol 2010; 34: 365-372.

23. Cook JL, Malliaras P, De Luca J, Ptasznik R, Morris ME, Goldie P: Neovascularization and pain in abnormal patellar tendons of active jumping athletes. Clin J Sport Med 2004; 14: 296-299.

24. van Snellenberg W, Wiley JP, Brunet G: Achilles tendon pain intensity and level of neovascularization in athletes as determined by color Doppler ultrasound. Scand J Med Sci Sports 2007; 17: 530-534.

25. O'Brien M: The anatomy of the Achilles tendon. Foot Ankle Clin 2005; 10: 225-238. 